\title{
Assessment of WHOQOL-old death and dying dimension in older adults residing in long-term care facilities in Brazil
}

\begin{abstract}
This study aimed to determine which factors are associated with scores on the Death and Dying dimension of The World Health Organization Quality of Life-OLD (WHOQOL-old) questionnaire in a population of older adults living in Long-term Care Facilities (LTCF) in Brazil. This was a representative cross sectional study conducted in Brazil. The sample was comprised of 127 older adults, aged 60-80+ who resided in long-term care facilities at the time of data collection. The World Health Organization Quality of Life-OLD questionnaire was used to assess the quality of life across various dimensions. Sociodemographic variables (sex, age, marital status, educational background) and tools including the Geriatric Depression Scale (GDS), Timed Up and Go (TUG), Katz Index of Independence, and Dynamometer grip strength were investigated as variables. Statistical analyses of T-test and ANOVA were used to compare the means. The Death and Dying dimension yielded the lowest mean on the WHOQOL-old. None of the exploratory variables investigated had a statistically significant relationship with the Death and Dying dimension, despite GDS scores being inversely correlated with scores on the WHOQOL-old overall $(p<0.001)$. Low scores on the Death and Dying dimension stress the need for care to incorporate emotional preparedness for death in LTCF to enhance the quality of life.
\end{abstract}

Keywords: death, long-term care, older adults, quality of life, aging
Volume 4 Issue 6 - 2019

\author{
Marina Celly Martins Ribeiro de Souza,' \\ Carolina Marques Borges,' Klara Bieniasz, ${ }^{2}$ \\ Anna Kellaher, ${ }^{2}$ Justine Wilson, ${ }^{2}$ Natália de \\ Cássia Horta, ${ }^{3}$ Tatiana Teixeira Barral de \\ Lacerda, ${ }^{3}$ Karla Geovani Marcelino ${ }^{4}$ \\ 'Associate Professor, Department of Public Health, The College \\ of New Jersey, USA \\ 2Undergraduate Student, The College of New Jersey, USA \\ ${ }^{3}$ Associate Professor, Department of Medicine- Pontifical \\ Catholic University of Minas Gerais, Brazil \\ ${ }^{4}$ Social Worker, Pontifical Catholic University of Minas Gerais, \\ Brazil
}

Correspondence: Marina Celly Martins Ribeiro de Souza, Associate Professor, Department of Public Health, The College of New Jersey, USA, Tel 609-77I-2232, ORCIDhttps://orcid. org/0000-0002-3503-4038, Email desouzam@tcnj.edu

Received: October 25, 2019 | Published: November 08, 2019

\section{Introduction}

Rising life expectancy and decreasing fertility rates have contributed to rapid aging among populations throughout the world. The population aged 60 and over is the fastest growing population, increasing at a rate of 3.26 percent per year globally. ${ }^{1}$ In Brazil, the population of older adults aged $60+$ is projected to triple by 2050 , which will subsequently leave the country with the fourth-largest older population in the world. ${ }^{1}$ Given this demographic shift and other factors including changes to family structure and roles, demand for long term care facilities (LTCF) will continue to significantly increase. In Brazil, the number of LTCF is on the rise, especially private LTCF in urban centers. ${ }^{2}$ Some studies have noted that with the aging population of Brazil, health systems in the nation should take a continuous and multidisciplinary organizational approach. ${ }^{3}$ Our study is an attempt to better understand the determinants of the quality of life (QOL) in older adults residing in LTCF, specifically in the Death and Dying dimension.

QOL is an important outcome of care for residents of long-term care facilities. The World Health Organization Quality of Life (WHOQOL) Group defines quality of life as an "individual's perception of their position in life in the context of the culture and value systems in which they live and in relation to their goals, expectations, standards, and concerns". ${ }^{4}$ To better understand the QOL of older adults, the World Health Organization developed a specific instrument called Quality of Life Instrument-Older Adults Module (WHOQOL-old) that takes into consideration dimensions of particular importance to older adults including Autonomy, Intimacy, and Death and Dying. ${ }^{5}$ The WHOQOL-old includes perceptions and feelings towards death and dying as a dimension measuring the quality of life. The attitudes of older adults regarding death and dying have been previously studied and show that the most prominent issues were found to be fear of the manner by which they would die, fear of becoming a burden to others, wanting to be able to prepare for death and having control over where they die, and issues related to assisted dying. ${ }^{6}$ These concerns underscore the complexity of factors which contribute to an older adult's attitude towards death.

Past literature has shown the effects of sociodemographic factors on quality of life among older adults using the WHOQOL-old and WHOQOL-bref, but previous studies lack a focus on the Death and Dying dimension of WHOQOL-old in a comparative analysis within a Brazilian population. ${ }^{7}$ A recent study found that there are approximately 84,000 older adults residing in 2072 LTCF in Brazil. ${ }^{8}$ It is important to understand what affects the quality of life of older adults living in these facilities, specifically with regards to attitudes addressed by this dimension.

This study aimed to determine associated factors with scores of the Death and Dying dimension of the WHOQOL-old among older adults living in LTCF in Brazil.

\section{Materials and methods}

This was a representative cross-sectional study conducted in Brazil. The representative sample was comprised of 127 older adults, 
aged 60-80+ who resided in long-term care facilities at the time of data collection. This is a population base representative cross-sectional study conducted in the Metropolitan Area of Belo Horizonte, which is the third largest urban agglomeration in Brazil. Simple random sampling was used in two stages: identifying and contacting all 170 LTCFs existing in the Metropolitan Area of Belo Horizonte, and assessing the total number of residents of the 156 LTCFs that agreed to be part of our study in the second stage, which comprised 625 individuals aged 60 years or older. From the 156 LTCFs that agreed to participate in the study, the sample was composed of 28 philanthropic and 16 private, with a total of 44 . Thus, considering the total of 625 older adults living in LTCFs, and therefore eligible to participate, and an estimation error of $10 \%$ and a $90 \%$ confidence level, a minimum sample of 119 individuals should be recruited.

The data collection was carried out in the period from December 2016 to June 2017 by 18 members of the research group after acceptance of the older adults in participating in the study through the signing of the Informed Consent Form and approval of the Brazilian National IRB (Approval Notice no. 1.873.609/2014). Prior to the application of the instruments, a lottery was carried out among the older adults in all of the 44 LTCFs, and then a cognitive screening instrument, the Mini-Mental State Exam (MMSE), was applied to the randomized participants as an inclusion criteria. In situations where the minimum score was not reached, another participant was drawn until the sample calculation was surpassed. A total of 127 older adults were deemed eligible for participation.

Scores on the Death and Dying dimension WHOQOL-old were collected from all participants and mean scores associated with the tested variables were analyzed using T-test and ANOVA to determine if there was a statistically significant difference of means. The studied sociodemographic variables were age, gender, marital status, years of schooling, time spent in the LTCF, Geriatric Depression Scale scores, Self perception of health, TUG (Timed Up and Go test) quartiles, Katz, and Dynamometer scores (Table 1).

Table I Sociodemographic distribution. $n=\mid 27$ older adults

\begin{tabular}{llll}
\hline $\begin{array}{l}\text { Variables } \\
\text { Categories }\end{array}$ & n & $\%$ \\
\hline Age Group (years) & $60-69$ & 41 & 32.3 \\
& $70-79$ & 47 & 37 \\
& $80-89$ & 31 & 24.4 \\
& $90+$ & 8 & 6.3 \\
Sex & Male & 65 & 51.2 \\
Farital Status & Female & 62 & 48.8 \\
& Single & 55 & 43.3 \\
& Married & 21 & 16.5 \\
& Widowed & 27 & 21.3 \\
Education (years formal & Separated/Divorced & 24 & 18.9 \\
Education) & I & 34 & 26.8 \\
& 2 & 79 & 62.2 \\
& 3 & 6.3 & 4.7 \\
\hline
\end{tabular}

Table Continued...

\begin{tabular}{llll}
\hline $\begin{array}{l}\text { Variables } \\
\text { Categories }\end{array}$ & n & $\%$ \\
\hline $\begin{array}{l}\text { Length of } \\
\text { institutionalization }\end{array}$ & I year or less & 43 & 33.9 \\
& 2 a 4 & 35 & 27.6 \\
& 5 or more & 49 & 38.6 \\
Nature of Admission at the LTCF & & \\
& Own Accord & 50 & 39.4 \\
& Family Promoted & 61 & 47 \\
& Abandoned & 6 & 4.7 \\
Negative Experience & Violence & I & 0.8 \\
$\begin{array}{l}\text { Activities Offered by } \\
\text { the LTCF }\end{array}$ & Required Participation & 91 & 71.7 \\
& $\begin{array}{l}\text { Suggested } \\
\text { Participation }\end{array}$ & 92 & 72.4 \\
& Individual Activities & 72 & 56.7 \\
\hline
\end{tabular}

\section{Instruments and variables}

\section{Quality of life (death and dying dimension)}

Quality of Life with regards to the Death and Dying dimension was assessed with the administration of the World Health Organization Quality of Life-old (WHOQOL-old) questionnaire. The WHOQOLold consists of 24 questions divided into six facets, forming additional domains that account for specific issues related to aging. The additional facets are: Functioning of the Senses, Autonomy, Past, Present and Future Activities, Social Participation, Death and Dying, and Intimacy ${ }^{4}$. The higher the score, the higher the quality of life.

\section{Self-perception of health}

A measure of the health status used since 1950 in gerontological research is the self-perception of health, presenting as a mediating role between human biology and psychology, being related to functional decline and being pointed as a strong indicator of mortality 9 . To determine self-rated health, the individual is asked how he rates his health in the last few days and gives him the choice of answer in five alternatives: very bad, bad, fair, good, and very good. Thus, selfperception of health encompasses an interpretative process to which the individual can encompass biological, psychosocial and social conditions, that is, any information that the individual recognizes as pertinent to the state of health. Therefore, it is a subjective measure, being a general survey referring to all the dimensions of health pointed out by the evaluated individuals. It stands out as a relevant outcome for gerontological research, since it is simple, short and global in scope. ${ }^{9}$

\section{Depression}

The Geriatric Depression Scale (GDS) was used to detect depressive symptoms in the older adult population. Originally developed in the English language by Yesavage and Brink ${ }^{10}$ the scale consisted of 30 items. In 1986, a reduced version with 15 items was elaborated by Sheikh and Yesavage, ${ }^{11}$ in clinical practice this version is interesting, since there is less time required for administration. It was then translated and adapted for the Brazilian population in 1999, by Almeida and Almeida where it obtained a cut-off point 5/6, 
sensitivity of $85.4 \%$ and specificity of $73.9 \% .^{12}$ The scale provides wide acceptance in a clinical and research environment, presenting an easy-to-understand methodology, answer in dichotomous format (yes/ no), ability to be self-applied or applied by an examiner, efficiency and low cost, and if properly employed, can become a valuable public health tool in the tracking and control of these conditions in nonspecialized environments. ${ }^{10}$ The GDS scores subjects on a scale of $0-15$. A score of 0-4 is considered not depressed, 5-8 indicates mild depression, 9-11 indicates moderate depression, and 12-15 indicates severe depression. ${ }^{13}$

\section{TUG, katz, dynamometer}

The Timed Up and Go test (TUG) is used as a screening tool in clinical settings to identify the risk of falling which patients have and determines an individual's dynamic and static balance and overall mobility. The activities in the TUG exam include rising from a seated chair, walking three meters, turning around, and retiring back to the seated position. This exam determines if a person may require a cane, walker, or other mobility aids. The Katz index assesses the functional status of individuals with regards to their capacity to independently perform daily living activities such as bathing, dressing, toiletting, transferring, continence, and feeding. The Dynamometer grip strength test shows the ability of an individual to exert force and shows overall arm strength. The grip strength test is used to determine hand function, physical status, and task demand. With age, some grip strength is expected to be lost with trends of decreasing muscle mass. All of these tests were performed to determine the overall mobility of the group, as mobility is a contributing factor to independence, and a person's lack of mobility may contribute to feelings of depression. ${ }^{14}$

\section{Results}

This study aimed to determine associated factors with scores of the Death and Dying dimension of the WHOQOL-old among older adults living in long-term care facilities in Brazil. The Death and Dying dimension yielded the lowest scores out of all of the WHOQOL-old dimensions. The sociodemographic characteristics distributions do not show any obvious contributions to the Death and Dying dimension (Table 1). There was no statistically significant relationship $(\mathrm{p}<0.05)$ between any of the six sociodemographic variables studied: age, gender, marital status, schooling, time, and health status, and scores on the Death and Dying dimension (Table 2). Likewise, no significant correlation was reported between the Death and Dying dimension and GDS scores $(\mathrm{p}=0.152)$.

Tests scores derived by the Geriatric Depression Scale (GDS), Katz Index of Independence in Activities of Daily Living, Timed Up and Go Test (TUG), and dynamometer grip strength, were not associated with scores on the Death and Dying dimension (Table 2).

Table 2 WHOQOL dimension death and dying. none of the variables studied had a statistically significant impact on scores in the death and dying dimension

\begin{tabular}{|c|c|c|c|c|c|}
\hline Variables & & Mean & Standard Deviation & Median & p-value \\
\hline \multirow[t]{4}{*}{ Age (years) } & $60-69$ & 30.2 & 26.8 & 18.8 & \\
\hline & $70-79$ & 36.4 & 31.1 & 31.1 & 0.085 \\
\hline & $80-89$ & 21.2 & 23.4 & 23.4 & \\
\hline & $90+$ & 19.5 & 27.4 & 27.4 & \\
\hline \multirow[t]{2}{*}{ Gender } & Male & 28.6 & 27.3 & 18.8 & 0.664 \\
\hline & Female & 30.7 & 29.2 & 18.8 & \\
\hline \multirow[t]{4}{*}{ Marital status } & Single & 27.2 & 26.2 & 18.8 & \\
\hline & Married & 37.5 & 33.1 & 43.8 & 0.478 \\
\hline & Widowed & 31.5 & 30.6 & 25 & \\
\hline & Divorced/Separated & 26.3 & 25.1 & 18.8 & \\
\hline \multirow[t]{4}{*}{ Schooling (years) } & 1 & 38.6 & 29.1 & 31.3 & \\
\hline & 2 & 26.8 & 27 & 18.8 & 0.182 \\
\hline & 3 & 23.4 & 33.5 & 9.4 & \\
\hline & 4 & 24 & 26.3 & 15.6 & \\
\hline \multirow[t]{3}{*}{ Length of institutionalization (years) } & $>1$ & 32.8 & 30.6 & 31.3 & \\
\hline & 02-Apr & 27.3 & 26.4 & 18.8 & 0.152 \\
\hline & $5+$ & 28.4 & 27.5 & 18.8 & \\
\hline \multirow[t]{3}{*}{ GDS } & $0-5$ & 25.2 & 28.5 & 12.5 & 0.152 \\
\hline & 06-Oct & 35.2 & 28.6 & 31.3 & \\
\hline & Nov-15 & 34.2 & 24 & 25 & \\
\hline \multirow[t]{2}{*}{ Self Perception of Health } & Great/Good & 32.1 & 31 & 18.8 & 0.294 \\
\hline & Poor/Very Poor & 26.8 & 24.5 & 18.8 & \\
\hline
\end{tabular}


Table Continued...

\begin{tabular}{|c|c|c|c|c|c|}
\hline Variables & & Mean & Standard Deviation & Median & p- value \\
\hline \multirow[t]{4}{*}{ TUG (Quartiles) } & Ist & 30.3 & 29.6 & 18.8 & 0.99 \\
\hline & 2nd & 28.1 & 28.7 & 21.9 & \\
\hline & $3 r d$ & 27.1 & 28.3 & 15.6 & \\
\hline & 4th & 28.1 & 32.9 & 18.8 & \\
\hline \multirow[t]{3}{*}{ Katz } & Very dependent & 27.4 & 27.9 & 18.8 & \\
\hline & Moderate dependence & 27.7 & 29.5 & 18.8 & 0.111 \\
\hline & Independence & 42.1 & 25.8 & 43.8 & \\
\hline \multirow[t]{3}{*}{ Dynamometer } & $<25 \%$ & 31 & 28.6 & 21.9 & \\
\hline & $25-50 \%$ & 27.7 & 28.7 & 18.8 & 0.862 \\
\hline & $>75 \%$ & 29.7 & 26.3 & 18.8 & \\
\hline
\end{tabular}

\section{Discussion}

The tested variables allowed to determine which sociological, psychological, and physical factors do not significantly contribute to low quality of life with regards to the Death and Dying dimension, despite the intuitive expectation that they should have an impact in the population of institutionalized older adults in Brazil.

Our study found that none of the sociodemographic variables studied had a statistically significant impact on scores in the Death and Dying dimension (Table 2). Contrarily, previous literature has found relationships between sociodemographic factors and scores on the Death and Dying dimension. Vitorino et al. ${ }^{14}$ found that men scored significantly higher than women on the Death and Dying dimension $(p=0.002)$ in a study of older adults in LTCF in Brazil. In a study of older adults in Oslo ${ }^{15}$, similarly the authors found that higher scores in the Death and Dying domain were associated with high age and male gender. Dragomirecká ${ }^{15}$ found that among older adults in Prague, low education and female gender were associated with lower scores in the Death and Dying dimension. The existence of these relationships between sociodemographic variables and the scores on the Death and Dying dimension within other populations may suggest that cultural factors are relevant with regards to the nature of the impact of these variables.

Our hypothesis that scores on the Death and Dying dimension would be inversely correlated with indicators of adverse physical and psychological outcomes was not supported by the results of our study. Given that the Death and Dying dimension is affected by individuals' fears and anxieties of dying, it is expected that depression would exacerbate such worries in older adults. This finding is especially surprising in the case of an absence of correlation with depression as measured by the Geriatric Depression Scale. For instance, a study in a population of German ${ }^{16}$ adults over the age of 60 found that out of ten sociodemographic variables, depressive symptoms measured by the GDS, have the strongest effect on all six dimensions of the WHOQOLold including Death and Dying, as well as the overall WHOQOLold score. In a cross-cultural study of older adults in six European countries, depression was identified as the strongest predictor for most quality of life domains. ${ }^{15}$ The previous mentioned study also found that a low GDS score was related to higher quality of life in the Death and Dying domain among older adults in Oslo, Leipzig, and Umea. ${ }^{15}$ Our finding of no significant correlation between the Death and Dying dimension and GDS scores contrasts these studies.
Nevertheless, literature exists that is concurrent with our finding. Schestatsky et al. ${ }^{17}$ did not find a significant correlation between GDS scores and scores in the Death and Dying dimension among older adults with Parkinson's disease in Brazil. However, it is important to note that the population with Parkinson's disease is not the same as residents in LTCFs, as older adults with Parkinson's face debilitating issues.

The lack of a significant relationship between the Death and Dying dimension and a GDS scores and sociodemographic factors indicates the presence of a confounding variable contributing to the insignificance of the Death and Dying dimension that not investigated in our study. The Death and Dying dimension of the WHOQOL-old evaluates attitudes an individual has including concerns regarding the way in which one will die, fear of not being able to control one's own death, fear of dying itself, and fear of a painful death. One potential explanation for low scores in the Death and Dying dimension among LTCF residents is a lack of formal training in palliative care among LTCF staff. Low mean scores on the Death and Dying dimension stress the need for care to incorporate emotional preparedness for death in LTCFs to enhance the quality of life. A study by Brazil..$^{18}$ surveyed four LTCF and found that staff performed poorly on the Palliative Care Quiz for Nurses (PCQN), a 20-item test used to measure nurses' knowledge in palliative care. A low understanding of the philosophy of palliative care among LTCF staff may impact residents' attitudes towards death and dying.

Another potential contributing factor is religion and spirituality, which was a sociodemographic factor that was not investigated by this study. A 2016 study of institutionalized older adults in Brazil studied the relationship between spiritual and religious coping strategies and quality of life. ${ }^{19}$ Negative strategies were significantly correlated with low scores in the Death and Dying dimension. ${ }^{19}$ The effects of religion, social support, living arrangements and education of caregivers should be considered in future research.

This study reflects the cultures and societal norms of its scenario as it was conducted in Brazil, and hence this data may not be congruent with findings in other countries, as these differences may affect the way LTCFs function in each country. With regards to implications with older adult populations, this study only questioned older adults in LTCF, and may not be useful in analyzing non-institutionalized older adults. Rural residents may have a different experience, due to differences in resources availability as well. As this was a cross- 
sectional study, we did not determine causality of any one factor, but effectively showed lack of correlation of the investigated determinants and quality of life outcomes for residents of LTCFs.

Ultimately, this study did not find an association between any of the sociodemographic variables observed or tests performed with the Death and Dying dimension. This lack of association can be related to the small sample size and indicates a need for further research in the aging population of Brazil, especially those living in LTCFs.

\section{Acknowledgements}

None.

\section{Conflicts of interest}

The authors declare to have no conflict of interest.

\section{References}

1. https://esa.un.org/unpd/wpp/publications/files/key_findings_wpp_2015. pdf.

2. Lacerda TTBD, Horta NDC, Souza MC, et al. Characterization of long-term care facilities for the elderly in the metropolitan region of Belo Horizonte. Revista Brasileira de Geriatria e Gerontologia. 2017;20(6):743-753.

3. Miranda GMD, Mendes ADCG, Silva ALAD. Population aging in Brazil current and future social challenges and consequences. Revista Brasileira de Geriatria e Gerontologia. 2016;19(3):507-519.

4. Development of the World Health Organization WHOQOL-BREF quality of life assessment. The WHOQOL Group. Psychol Med. 1998;28(3):55158.

5. Peel NM, Bartlett HP, Marshall AL. Measuring quality of life in older people: reliability and validity of WHOQOL-OLD. Australasian Journal on Ageing. 2007.

6. Lloyd-Williams M, Kennedy V, Sixsmith A, et al. The end of life aqualitative study of the perceptions of people over the age of 80 on issues surrounding death and dying. Journal of pain and symptom management. 2007;34(1):60-66.

7. Gobbens RJ, Remmen R. The effects of sociodemographic factors on quality of life among people aged 50 years or older are not unequivocal: comparing SF-12, WHOQOL-BREF, and WHOQOL-OLD. Clinical interventions in aging. 2019;14:231-239.
8. Paiva MHPD, Pegorari MS, Nascimento JS. et al. Factors associated with quality of life among the elderly in the community of the southern triangle macro-region, Minas Gerais, Brazil. Ciencia \& saude coletiva. 2016;21(11):3347-3356.

9. Jylhä M, Guralnik JM, Ferucci L, et al. Is self-rated health comparable across cultures and genders?. J Gerontol Psych Sci Soc Sci. 1998;53B:S144-S152.

10. Yesavage JA, Brink TL, Rose TL. et al. Development and validation of a geriatric depression screening scale: A preliminary report. Journal of Psychiatric Research. 1992-1993;17(1):37-49.

11. Sheikh JI, Yesavage JA. Geriatric Depression Scale (GDS): Recent evidence and development of a shorter version. Clinical Gerontologist: The Journal of Aging and Mental Health. 1986;5(1-2):165-173.

12. Almeida OP, Shirley A. Confiabilidade da versão brasileira da Escala de depressão em geriatria (GDS) versão reduzida. Arq Neuro-Psiquiatr. 1999;57(2B):421-426.

13. Xiao H, Yoon JY, Bowers B. Living arrangements and quality of life: mediation by physical function and depression. Western journal of nursing research. 2016;38(6):738-752.

14. Vitorino LM, Paskulin LM, Viann LA. Quality of life among older adult residents in long-stay care facilities. Revista Latino-Americana de Enfermagem. 2012;20(6).

15. Dragomirecka E, Bartonova J, Eisemann M, et al. Demographic and psychosocial correlates of life in the elderly from a crosscultural perspective. Clinical Psychology \& Psychotherapy Journal. 2008;15(3):193-204.

16. Conrad I1, Matschinger H, Riedel-Heller S, et al. The psychometric properties of the German version of the WHOQOL-OLD in the German population aged 60 and older. Health and quality of life outcome. 2014;12(105).

17. Schestatsky P1, Zanatto VC, Margis R, et al. Quality of life in a Brazilian sample of patients with Parkinson's disease and their caregivers. Brazilian Journal of Psychology. 2006;28(3):209-211.

18. Brazil K1, Brink P, Kaasalainen S, et al. Knowledge and perceived competence among nurses caring for the dying in long-term care homes. Int J Palliat Nurs. 2012;18(2):77-83.

19. Vitorino LM, Lucchetti G, Santos AE, et al. Spiritual religious coping is associated with quality of life in older adults. Journal of Religion and Health. 2016;55(2):549-559. 